\title{
Análise de Crescimento de Cultivares de Panicum maximum Jacq. ${ }^{1}$
}

\author{
Carlos Augusto de Miranda Gomide 2 , José Alberto Gomide ${ }^{3}$
}

\begin{abstract}
RESUMO - O objetivo deste trabalho foi analisar o crescimento de três cultivares de Panicum maximum: Mombaça, Tanzânia e Vencedor cultivados em vasos, sob cobertura de lona plástica transparente. Os vasos foram cheios com $6 \mathrm{dm}^{3} \mathrm{de}$ latossolo vermelhoamarelo, textura argilosa, contendo alto teor de matéria orgânica. O solo, após ser peneirado, recebeu calagem e enriquecimento com $\mathrm{N}$ e P, $150 \mathrm{mg} / \mathrm{dm}^{3}$ de cada elemento. As sementes foram plantadas em vasos, num total de 54 . Após desbaste, cinco plantas foram deixadas por vaso. Diariamente os vasos foram molhados e quinzenalmente fertilizados, cada um, com $50 \mathrm{mg} / \mathrm{dm}^{3} \mathrm{de} \mathrm{N} \mathrm{e} \mathrm{K,} \mathrm{em} \mathrm{solução} \mathrm{aquosa.}$ Cada cultivar foi colhido às idades de 17, 24, 31, 38, 45 e 52 dias após emergência da semente. Houve três repetições para cada cultivar e idade. Em cada colheita, as plantas foram cortadas próximo à superfície do solo e separadas em folhas, colmo, raízes e material morto. A área das lâminas foliares foi medida em integrador de área foliar. Lâminas foliares, colmo e sistema radicular foram pesados após secagem a $60^{\circ} \mathrm{C}$ por 72 horas. Valores instantâneos foram estimados para os índices de crescimento: área foliar específica (AFE), razão de peso foliar (RPF), razão de área foliar (RAF), taxa assimilatória líquida (TAL) e taxa de crescimento relativo (TCR), de todos três cultivares. Houve diferenças entre os cultivares quanto aos valores instantâneos de RAF e TCR à idade de 17 dias. As variações de AFE foram as principais causas das mudanças em RAF. Nenhuma diferença foi observada entre os cultivares quanto à TAL.
\end{abstract}

Palavras-chave: área foliar específica, razão de área foliar, razão de peso foliar, taxa assimilatória líquida, taxa de crescimento relativo

\section{Growth Analysis of Panicum Maximum Jacq. Cultivars}

\begin{abstract}
The objective of this work was to analyze the growth of three cultivars of Panicum maximum: Mombaça, Tanzânia and Vencedor grown in pots, under a transparent plastic cover. The pots were filled with $6 \mathrm{dm}^{3}$ of redyellow latossol, clayish texture, with high organic matter content. The soil, after sifted, was limed and enriched with $\mathrm{N}$ and $\mathrm{P}, 150 \mathrm{mg} / \mathrm{dm} \mathrm{m}^{3}$ of each element. The seeds were seeded in the pots, in a total of 54. After thinning, five seedlings were left per pot. The pots were daily watered and fortnightly fertilized, each one, with $50 \mathrm{mg} / \mathrm{dm}^{3} \mathrm{~N}$ and $\mathrm{K}$, in aqueous solution. Each cultivar was harvested at the ages of 17, 24, 31, 38, 44 and 52 days after seedlings emergence. There were three replicates for each cultivar and age. In each harvest, the plants were clipped close to the soil surface, and separated into: leaf blades, stem, root system and dead material. The leaf blade area was measured with a digital area meter system. The leaf blade, stem, and root system were weighed after drying at $60^{\circ} \mathrm{C}$ for 72 hours. Instantaneous values were estimated for the growth indices: specific leaf area (SLA), leaf weight ratio (LWR), leaf area ratio (LAR), net assimilation rate (NAR) and relative growth rate (RGR), of all cultivars. There were differences among cultivars as for the instantaneous values of LAR and RGR at the age of 17 days. Variations of the SLA were the mainly causes in the changes in the LAR. No difference was observed among cultivars for NAR.
\end{abstract}

Key Words: leaf area ratio, leaf weight ratio, net assimilation rate, relative growth rate, specific leaf area

\section{Introdução}

O crescimento das plantas superiores fundamenta-se na conversão da energia luminosa em energia química, cuja intensidade é proporcionalà intercepção e captura da luz pelo dossel da cultura. Daí a importância das estimativas dos índices: razão de área foliar (RAF) e índice de área foliar (IAF), que representam os componentes morfológicos da taxa de crescimento relativo (TCR) e taxa de crescimento cultural (TCC), respectivamente, que têm a taxa de assimilação líquida (TAL) como componente fisiológico (LAMBERS, 1987), visto que:

\section{$\mathrm{TCR}=\mathrm{TAL} X \mathrm{RAF}$ \\ $\mathrm{TCC}=$ TAL X IAF}

À análise de crescimento vegetal, pelo método clássico, interessam as estimativas dos índices: 1 razão de área foliar (RAF), área foliar por unidade de peso de toda a planta $\left(\mathrm{m}^{2} / \mathrm{g}\right) ; 2$ - área foliar específica (AFE), área foliar por unidade de peso das folhas $\left(\mathrm{m}^{2} / \mathrm{g}\right) ; 3$ - razão de peso foliar (RPF), peso das folhas por unidade de peso da planta inteira $(\mathrm{g} / \mathrm{g}) ; 4$ - taxa de crescimento relativo (TCR), incremento de peso por unidade de peso da planta inteira por unidade de tempo, geralmente diário ou semanal, (g/g·dia oug/g semana);

\footnotetext{
${ }^{1}$ Parte da Tese de MS do primeiro autor, pesquisa financiada pela FAPEMIG.

${ }^{2}$ Estudante de Mestrado do DZO-UFV.

${ }^{3}$ Pesquisador IA do CNPq.
} 
e 5 - taxa de crescimento cultural (TCC) - incremento diário ou semanal de peso por unidade de área de solo ( $\mathrm{g} / \mathrm{m}^{2}$ dia ou $\mathrm{g} / \mathrm{m}^{2} \cdot$ semana $)$.

Freqüentemente, para determinada condição de meio, o rendimento forrageiro mostra melhor correlação com as características da planta (RAF) ou do relvado (IAF) associadas à intercepção de luz do que com a eficiência fotossintética da planta (LAWLOR, 1995). Para BEADLE (1993), a TCC varia mais em função do IAF que em função da TAL. POORTER (1989) relata forte correlação negativa entre TAL e RAF, sendo este último o principal determinante da TCR.

O balanço fotossíntese - respiração determina a quantidade de assimilado disponível para o crescimento vegetal - a TAL. Entretanto, a alocação preferencial de carbono pelas diferentes frações da planta folhas, colmo e sistema radicular -, condicionada pelo meio e/ou manejo, determina variações nos índices de crescimento AFE, RPF, RAF, IAF. Assim, as estimativas destes índices podem fornecer subsídios para o entendimento das adaptações experimentadas pelas plantas sob as diferentes condições de meio: luz, temperatura, umidade e fertilidade do solo. Assim, a análise de crescimento auxilia a interpretação das diferenças entre genótipos e de suas adaptações ao meio em que são cultivados e ao manejo.

Fatores do meio, principalmente luz e temperatura, condicionam os valores dos índices de crescimento vegetal. Altas temperaturas favorecem a rápida expansão de folhas delgadas e, conseqüentemente, favorecem a RAF, via incremento da AFE. Segundo ROBSON et al. (1988), a elevação da temperatura tem pouco efeito sobre a RAF e menos ainda sobre a TAL. Em azevém perene (Lolium perene L.), a TAL respondeu linearmente à intensidade luminosa, mas mostrou pequena resposta à variação térmica (SILSBURY, 1971). Enquanto a TAL varia proporcionalmente, a RAF varia inversamente com a radiação; assim, o efeito positivo da radiação sobre a TCR é atribuído à sua influência sobre a TAL (ROBSON et al., 1988).

Maior alocação de assimilados para a síntese de folhas resulta em maiores valores de RPF e, conseqüentemente, aumento da RAF, que, todavia, também pode advir de elevação da $\operatorname{AFE}(R A F=R P F X$ $A F E$ ) em resposta à adaptação da planta à condição de baixa luminosidade ou baixa fertilidade do solo (POORTER, 1989).

Adaptações morfofisiológicas foram observadas em capim-colonião (Panicum maximum) em resposta à redução da intensidade luminosa. Neste estudo, incre- mento da AFE e RAF preveniram o comprometimento da TCR da gramínea (DIAS FILHO et al., 1996).

A taxa de assimilação líquida varia em função do genótipo e dos fatores luz e temperatura, em razão dos efeitos destes fatores sobre a eficiência fotossintética das folhas e a respiração vegetal. As perdas respiratórias podem alcançar de 35 a $45 \%$ (PARSONS et al., 1983b; ROBSON, 1973) e até $60 \%$ (KRAUSS et al., 1989) do carbono fixado. Segundo PARSONS e PENNING (1988), 50\% do carbono fixado são consumidos no crescimento do sistema radicular e na respiração da planta.

LAMBERS (1987) enfatiza como a TAL varia em função do balanço entre os processos de fotossíntese e respiração vegetal. Por outro lado, LAMBERS et al. (1989) enfatizam ainda a importância da magnitude do aparelho fotossintético relativamente ao restante da planta, como outra condição determinante das estimativas da TAL.

Em reconhecimento do papel das perdas respiratórias, KRAUSS et al. (1989) propõem a seleção de cultivares forrageiros cujas folhas adultas apresentem baixas taxas respiratórias; essas plantas poderiam ser manejadas segundo desfolhas menos intensas e/ou menos freqüentes. Genótipos com baixa taxa respiratória das folhas adultas, tendo menor custo com respiração de mantença, são, potencialmente, mais produtivos entre os que mantêm o mesmo número de folhas por perfilho (PILBEAM, 1992).

O presente estudo teve por objetivo interpretar possíveis diferenças de crescimento entre três cultivares de Panicum maximum.

\section{Material e Métodos}

O experimento foi conduzido em vasos, sob cobertura plástica transparente. Cada vaso, num total de 54 , com $25 \mathrm{~cm}$ de diâmetro, tinha capacidade para $6 \mathrm{dm}^{3}$ de solo. O solo utilizado, latossolo vermelhoamarelo, de textura argilosa, com alto teor de matéria orgânica, foi passado em peneira com malhas de 4 mm. Após calagem, segundo COMISSÃO DE FERTILIDADE DO SOLO DE MINAS GERAIS (1989), e adubação com $150 \mathrm{mg} / \mathrm{dm}^{3}$ de $\mathrm{P}$ e $\mathrm{N}$, o solo apresentou a seguinte composição química: $\mathrm{pH}$ em água, 6,$2 ; \mathrm{P}, 92,6 \mathrm{mg} / \mathrm{dm}^{3} ;$ Ca trocável, $3,1 \mathrm{cmol} / \mathrm{dm}^{3} ; \mathrm{Mg}$ trocável, $0,7 \mathrm{cmol} / \mathrm{dm}^{3}$; soma de bases, $4,07 \mathrm{cmol} / \mathrm{dm}^{3} ; \mathrm{H}$ $+\mathrm{Al}, 14,7 \mathrm{cmol} / \mathrm{dm}^{3}$; CTC, 18,77; Al trocável, 0,1 $\mathrm{cmol} / \mathrm{dm}^{3}$; saturação por bases, $21,7 \%$; e saturação por alumínio, $2,4 \%$.

Sementes dos cultivares Mombaça, Tanzânia e 
Vencedor de Panicum maximum foram semeadas nos vasos em 09/01/1996. Foram colocadas, em média, 30 sementes/vaso, de modo a obter cinco plantas por vaso, após desbaste, efetuado em $22 / 01 / 96$. Após a semeadura, os vasos foram irrigados diariamente com água de torneira. Quinzenalmente, procedeu-se à adubação à base de $50 \mathrm{mg} / \mathrm{dm}^{3}$ de $\mathrm{N}$ e K, aplicada em solução aquosa.

As condições de temperatura e radiação durante o período de ensaio foram registradas por uma miniestação meteorológica instalada sob a cobertura de plástico transparente (Figura 1).

Colheitas foram feitas às idades de 17,24,31,38, 45 e 52 dias após a emergência, entre 01/02 e 07/03/1996. O período experimental se estendeu de 09/01/1996 a 07/03/1996. Foram observadas três repetições segundo o delineamento de blocos completos casualizados. Ao tempo de colheita, em cada idade, as plantas foram cortadas rente à superfície do solo. Em laboratório procedeu-se à separação da parte aérea em lâminas foliares, colmos e material morto. As lâminas foliares de cada planta foram medidas em um sistema digital integrador de área.

Lâminas foliares e colmos de cada vaso foram secados até peso constante em estufa a $60^{\circ} \mathrm{C}$. Da mesma forma, procedeu-se ao sistema radicular de cada vaso, recuperado mediante lavagem sob água corrente sobre um conjunto de peneiras com malhas decrescentes.

Aos valores médios de peso seco total da planta, área e peso seco de folhas de cada cultivar foram ajustadas equações quadráticas, em função da idade, a fim de se estimarem os valores instantâneos de TCR, TAL e RAF conforme RADFORD (1967). A estes índices foi ajustada a equação $\mathrm{Y}=(\mathrm{a}+\mathrm{cx}) /(1+\mathrm{bx})$, em que $\mathrm{a}, \mathrm{b}$ e c são constantes, $\mathrm{a}$ fim de descrever a sua variação com a idade (x).

Os índices AFE e RPF foram estimados a partir dos valores observados para área foliar (A), peso foliar (Pf) e peso total da planta (Pp):

$$
\mathrm{AFE}=\mathrm{A} / \mathrm{Pf} \text { e } \mathrm{RPF}=\mathrm{Pf} / \mathrm{Pp}
$$

Todos os índices de crescimento foram analisados segundo o modelo:

em que

$$
\mathrm{Y}_{\mathrm{ijk}}=\mu+\mathrm{B}_{\mathrm{i}}+\mathrm{I}_{\mathrm{j}}+\mathrm{C}_{\mathrm{k}}+\mathrm{IC}_{\mathrm{jk}}+\mathrm{E}_{\mathrm{ijk}}
$$

$\mathrm{Y}_{\mathrm{ijk}}=$ observação relativa ao $\mathrm{k}_{\text {ésimo }}$ cultivar à $\mathrm{j}_{\text {ésima }}$ idade, no $\mathrm{i}_{\text {ésimo }}$ bloco;

$\mu \quad=$ média geral;

$\mathrm{B}_{\mathrm{i}}=$ efeito do $\mathrm{i}_{\text {ésimo }}$ bloco, $\mathrm{i}=1,2,3$ blocos;

$\mathrm{I}_{\mathrm{j}}=$ efeito da $\mathrm{j}_{\text {ésima }}$ idade, $\mathrm{j}=1,2,3,4,5,6$ idades; $\mathrm{C}_{\mathrm{k}}=$ efeito do $\mathrm{k}_{\text {ésimo }}$ cultivar, $\mathrm{k}=1,2,3$ cultivares;

$\mathrm{IC}_{\mathrm{jk}}=$ interação da $\mathrm{j}_{\text {ésima }}$ idade com o $\mathrm{k}_{\text {ésimo }}$ cultivar; $\mathrm{e}$

$\mathrm{E}_{\mathrm{ijk}}=$ erro experimental.

As médias foram testadas a $5 \%$ de probabilidade pelo teste Tukey.

\section{Resultados e Discussão}

Interação idade $\mathrm{x}$ cultivar foi observada para os valores de RAF, sendo observadas diferenças entre os cultivares $(\mathrm{P}<0,05)$ apenas aos 17 dias de idade. $\mathrm{O}$ cultivar Mombaça mostrou as mesmas estimativas de RAF ao longo do período experimental, enquanto os outros dois cultivares não exibiram variação da RAF após a idade de 24 dias (Figura 2).

A redução da RAF, principalmente no período de 17 a 24 dias de idade, sinaliza prejuízo para a economia de carbono das plantas, pois reflete progressiva diminuição do aparelho fotossintético relativamente ao crescente peso da planta, que representa maior custo da respiração de mantença (ROBSON, 1973).

As variações observadas podem decorrer de mudanças de RPF e/ou AFE, os dois componentes da RAF. Apesar da interação cultivar $x$ idade $(P<0,05)$ relativamente às estimativas de RPF, os valores observados para este componente da RAF (Tabela 1) não representam diferenças substanciais. Os cultivares não diferiram entre si, senão à idade de 17 dias, quando o Tanzânia exibiu menor valor (Tabela 1). A média geral de $0,436 \mathrm{~g} / \mathrm{g}$ evidencia a proporção, razoavelmente constante, do aparelho fotossintético relativamente à planta toda. Assim, a evidência é que as variações observadas para RAF decorreram dos valores de $\mathrm{AFE}$, que variaram linear e negativamente com a idade da planta: $\mathrm{Y}=0,062-0,0009 \mathrm{X} ; \mathrm{r}=-0,96$.

A redução nas estimativas de AFE durante o desenvolvimento das plantas na fase de estabelecimento reflete o acentuado crescimento ponderal das folhas relativamente à área foliar, indicando assim o aumento na espessura das folhas.

O mesmo padrão foi observado nos valores de AFE para cultivares de Cynodon dactylon apenas após o $28^{\circ}$ dia de rebrota, quando foi observado o valor máximo de $0,023 \mathrm{~m}^{2} / \mathrm{g}$ (GOMIDE, 1996). Entretanto, para os mesmos cultivares de Cynodon dactylon, divergências foram observadas quanto ao padrão e à magnitude dos valores de RPF, pois apresentam queda no transcorrer da rebrota e valores 3 a 4 vezes menores que os encontrados neste estudo. Os menores valores de RPF para Cynodon 
Tabela 1 - Razão de peso foliar (RPF) dos cultivares Mombaça, Tanzânia e Vencedor em diferentes idades

Table 1 - Leaf weight ratio (LWR) of Mombaça, Tanzânia and Vencedor at different ages

\begin{tabular}{lccc}
\hline \multirow{2}{*}{$\begin{array}{l}\text { Idade }(\mathrm{d}) \\
\text { Age }\end{array}$} & Mombaça & Tanzânia & Vencedor \\
\cline { 2 - 4 } 17 & $-0^{-}-{ }^{2}, 439^{\mathrm{Aa}}$ & $0,397^{\mathrm{Bb}}$ & $0,460^{\mathrm{Aa}}$ \\
24 & $0,440^{\mathrm{Aa}}$ & $0,420^{\mathrm{Aab}}$ & $0,436^{\mathrm{Aab}}$ \\
31 & $0,451^{\mathrm{Aa}}$ & $0,443^{\mathrm{Aa}}$ & $0,437^{\mathrm{Aab}}$ \\
38 & $0,460^{\mathrm{Aa}}$ & $0,458^{\mathrm{Aa}}$ & $0,434^{\mathrm{Aab}}$ \\
45 & $0,452^{\mathrm{Aa}}$ & $0,435^{\mathrm{Aa}}$ & $0,426^{\mathrm{Aab}}$ \\
52 & $0,445^{\mathrm{Aa}}$ & $0,423^{\mathrm{Aab}}$ & $0,396^{\mathrm{Bb}}$ \\
\hline
\end{tabular}

$\mathrm{A}>\mathrm{B}$ compara cultivares na mesma idade; $\mathrm{a}>\mathrm{b}$ compara as idades dentro de cultivares $(P<0,05)$.

$A>B$ compare cultivars in the same age; $a>b$ compare ages in the same cultivar $(P<.05)$.

justificam-se em razão, principalmente, de sua mais estreita relação folha/ colmo.

As estimativas de TAL não revelaram diferenças entre cultivares, nem interação cultivar x idade. Assim, o perfil da curva destas estimativas é apresentado em função média para os três cultivares (Figura 3), no qual se observam acentuada queda da TAL entre as idades de 17 e 24 dias do crescimento de estabelecimento e progressiva e suave redução nas idades subseqüentes. A estimativa de $32,8 \mathrm{~g} / \mathrm{m}^{2} \cdot$ dia à idade de 17 dias, aparentemente muito alta, decorre das características da área foliar: pequena e de alta capacidade fotossintética, própria de gramíneas tropicais, assim como das condições favoráveis de temperatura e radiação (Figura 1) e disponibilidade de água e nutrientes.

A acentuada diminuição entre 17 e 24 dias poderia ser entendida como conseqüência do acentuado incremento na área foliar, assim como de intensificação da respiração vegetal, visto não se esperar drástica perda da capacidade fotossintética das folhas nesta fase do desenvolvimento das plantas. De fato, a área foliar cresceu $330 \%$ entre 17 e 24 dias; $56 \%$ entre 24 e 31 dias; e $17 \%$ a cada semana subseqüente. GOMIDE e GOMIDE (1996) observaram que o perfilhamento destes cultivares ocorreu entre as idades de 7 e 21 dias. Por outro lado, a progressiva redução da TAL nas idades subseqüentes poderia ser explicada, em função de redução na capacidade fotossintética das folhas em expansão, em razão do progressivo sombreamento mútuo (WILSON e LUDLOW, 1970; WOLEDGE e LEAFE, 1976; e WOLEDGE, 1978), bem como da redução da eficiência fotossintética do dossel, em decorrência da avançada idade média de suas folhas (PARSONS et al., 1983a; WOLEDGE e LEAFE, 1976), além do progressivo aumento da carga respiratória de manutenção.

A semelhança entre as curvas de RAF e TAL merece ser realçada. Conforme já mencionado, a redução da RAF entre as idades de 17 e 24 dia, sinalizando acentuada elevação no custo da respiração de manutenção da planta, é consistente com simultânea queda da TAL, visto que este índice resulta do balanço na troca gasosa vegetal (LAMBERS, 1987; LAMBERS, et al., 1989).

Para as condições do presente estudo, o padrão observado na Figura 3 não pode ser atribuído à diminuição da radiação luminosa, cujos valores mais altos correspondem à fase final do ensaio (Figura 1).

Interação cultivar $\mathrm{x}$ idade $(\mathrm{P}<0,05)$ foi observada para as estimativas de TCR. Assim, o padrão de variação dessas estimativas é mostrado para cada cultivar (Figura 4). Apenas à idade de 17 dias verificaram-se diferenças entre os cultivares; por outro lado, o efeito da idade $(\mathrm{P}<0,05)$ só foi observado nos cultivares Tanzânia e Vencedor, em que mais elevados valores de TCR $(\mathrm{P}<0,05)$ correspondem à idade de 17 dias. As estimativas de 3,5 e 5,1 g/g dia, respectivamente, para Tanzânia e Vencedor, refletem o intenso crescimento da planta em sua fase inicial de estabelecimento: aparentemente muito elevado. Vale enfatizar que o presente trabalho apresenta valores instantâneos, enquanto mais freqüentemente são publicados valores médios relativos a longo período de tempo de crescimento. Esta é a estimativa média de $0,055 \mathrm{~g} / \mathrm{g} \cdot \mathrm{dia}$ para a TCR de feijão-fava (Vicia faba L.) encontrada por COSTA et al. (1997).

Conforme sugere a Figura 4, a estimativa da TCR, a partir da idade de 31 dias, foi inferior a $0,1 \mathrm{~g} / \mathrm{g} \cdot \mathrm{dia}$, para os três cultivares. De fato, a média dos valores observados entre as idades de 31 e 52 dias, para os três cultivares, foi de $0,05 \mathrm{~g} / \mathrm{g} \cdot \mathrm{dia}$, valor consistente com os encontrados para cultivares de Cynodon dactylon por GOMIDE (1996). Os valores médios de TCR relatados por esse autor, durante a rebrota de cultivares de Cynodon dactylon, mostraram-se constantes até a idade de 21 a 35 dias, quando se obteve valor máximo de $0,065 \mathrm{~g} / \mathrm{g} \cdot$ dia e posterior redução em idades mais avançadas da rebrota.

Visto que TCR $=$ RAF X TAL, a semelhança de padrão para estes três índices (Figuras 2, 3 e 4) sugere que, durante a fase de estabelecimento do presente estudo, tanto RAF como TAL tiveram igual influência sobre as estimativas de TCR observadas. Entretanto, em feijão-fava a variação na TCR de um 
Rev. bras. zootec.

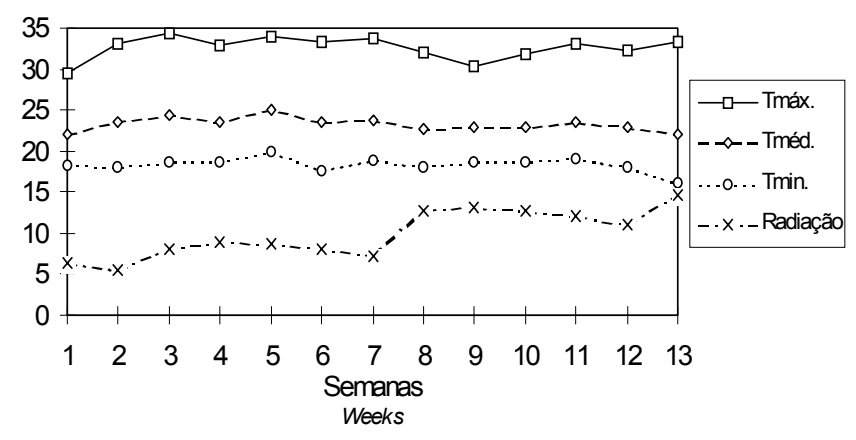

Figura 1 - Temperaturas máximas, médias e mínimas $\left({ }^{\circ} \mathrm{C}\right)$, média semanal e radiação total $\left(\mathrm{MJ} / \mathrm{m}^{2}\right.$.dia $)$ durante o período experimental.

Figure 1 - Maximum, medium and minimal temperatures $\left({ }^{\circ} \mathrm{C}\right)$, (weekly average) and total radiation $\left(\mathrm{MJ} / \mathrm{m}^{2}\right.$.day) during the experimental period.

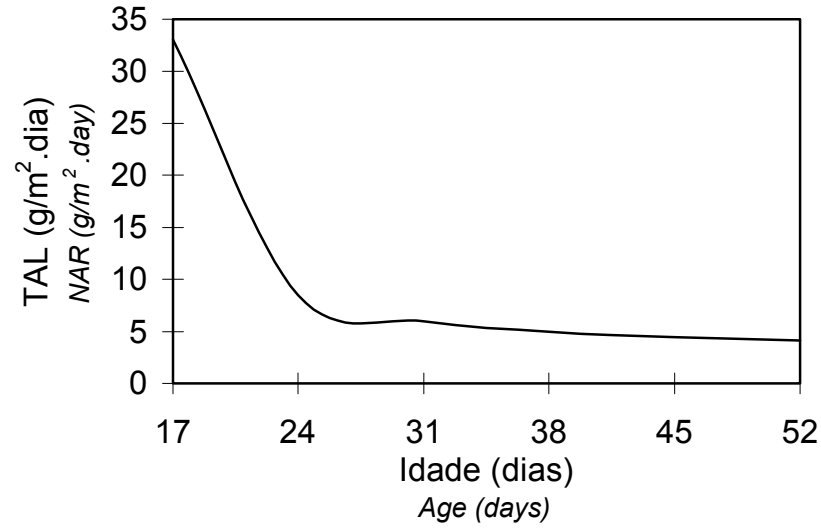

Figura 3 - Taxa assimilatória líquida (TAL) dos cultivares Mombaça, Tanzânia e Vencedor em diferentes idades.

Figure 3 - Net assimilation rate (NAR) of Mombaça, Tanzânia and Vencedor at different ages.

ano para outro foi mais dependente de TAL que RAF (COSTA et al., 1997). Porém, segundo POORTER et al. (1989), a RAF é o principal componente de TCR determinando sua variação.

\section{Conclusões}

Os cultivares não diferiram quanto à taxa assimilatória líquida, mas apenas quanto à razão de área foliar e taxa de crescimento relativo na fase

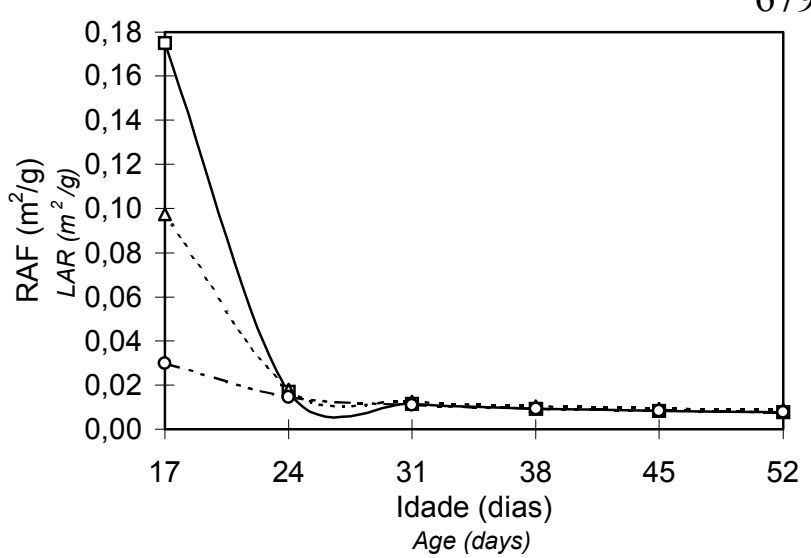

Figura 2 - Razão de área foliar (RAF) dos cultivares Mombaça (O), Tanzânia $(\Delta)$ e Vencedor ( $\square$ ) em diferentes idades.

Figure 2 - Values of leaf area ratio (LAR) of Mombaça (O), Tanzânia ( $\Delta$ ) and Vencedor (\) at different ages.

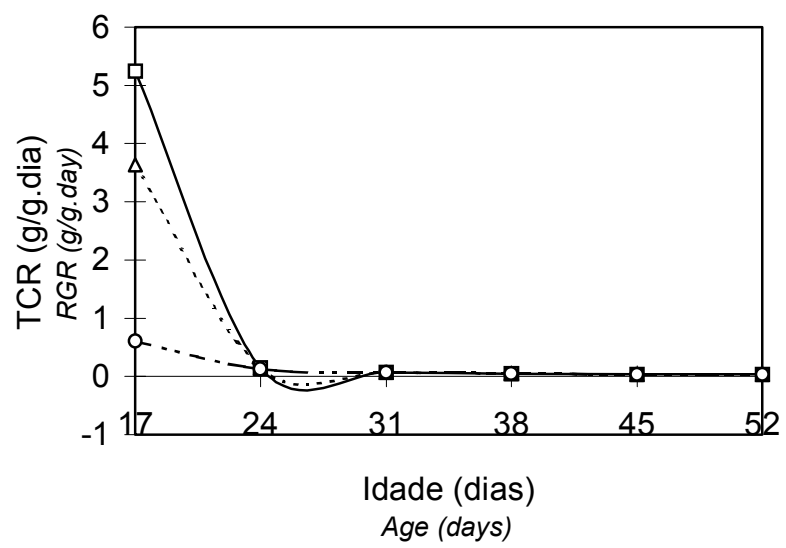

Figura 4 - Taxa de crescimento relativo (TCR) dos cultivares Mombaça (O), Tanzânia $(\Delta)$ e Vencedor (ㅁ) em diferentes idades.

Figure 4 - Relative growth rate (RGR) of Mombaça (O), Tanzânia $(\Delta)$ and Vencedor ( 1 ) at different ages.

inicial de estabelecimento. Posteriormente, os cultivares eqüivaleram-se quanto aos valores instantâneos desses índices de crescimento. O padrão de variação desses índices revelou elevados valores nas primeiras semanas, exibindo acentuada queda entre a terceira e quinta semana e progressiva redução tendendo à estabilidade posteriormente. Assim, os resultados obtidos não evidenciam diferença de potencial de rendimento forrageiro entre os cultivares durante sua fase produtiva. 


\section{Referências Bibliográficas}

BEADLE, C.L. 1993. Growth analysis. In: HALL, D.O., BOLHARNORDENKAMPF, H.R., LEE GOOD, R.C. et al. Photosynthesis and production in a changing environment: a field and laboratory manual. London: England. p.36-46.

COSTA, L.C., MORISON, J., DENNETT, M. 1997. Effects of the weather on growth and radiation intercepted by Faba bean. Pesq. Agrop. Bras., 32(3):277-281.

COMISSÃO DE FERTILIDADE DO SOLO DO ESTADO DE MINAS GERAIS. 1989. Recomendações para o uso de corretivos e fertilizantes em Minas Gerais: $4^{a}$ aproximação. Lavras. 159p.

DIAS FILHO, M.B., CHAGAS JR., A.F., CARVALHO, C.J.R. Respostas morfológicas de Panicum maximum a diferentes condições de luminosidade. In: REUNIÃO ANUAL DA SOCIEDADE BRASILEIRA DE ZOOTECNIA, 33, 1996, Fortaleza. Anais... Fortaleza: SBZ, 1996, p.62-64.

GOMIDE, C.A.M., GOMIDE, J.A. Morfogênese e análise de crescimento de cultivares de Panicum maximum. In: REUNIÃO ANUAL DA SOCIEDADE BRASILEIRA DE ZOOTECNIA, 33, 1996, Fortaleza. Anais... Fortaleza: SBZ, 1996, p.403-405.

GOMIDE, C.C.C. Algumas características morfológicas e químicas de cinco cultivares de Cynodon. Jaboticabal: UNESP, 1996. 100p. Dissertação (Mestrado em Zootecnia) - Universidade Estadual de São Paulo, 1996.

KRAUS, E., WILSON, D., ROBSON, M.J. et al. 1989. Respiration: correlation with growth rate and its quantitative significance for net assimilation rate and biomass production. In: Lambers, H. et al. Causes and consequences of variation in growth rate and productivity of higher plants. Hague: Netherlands. p.187-198.

LAMBERS, H. 1987. Does variation in photosynthetic rate explain variation in growth rate? Netherlands J. Agric. Sci.,35:505-519.

LAMBERS, H., FREIDJSEN, N. POORTER, H. et al. 1989. Analysis of growth based on net assimilation rate and nitrogen productivity. Their physiological background. In: Lambers, $\mathrm{H}$. et al. Causes and consequences of variation in growth rate and productivity of higher plants. Hague: Netherlands. p.1-17.

LAWLOR, D.W. 1995. Photosynthesis, productivity and environment. J. Exp. Botany, 46:1449-1461(special issue)

PARSONS, A., LEAFE, E.L., COLLETT, B. et al. 1983a. The physiology of grass production under grazing. I Characteristics of leaf and canopy photosynthesis of continuously grazed sward. J. Appl. Ecology, 20(1):117-126.

PARSONS, A.J., LEAFE, E.L., COLLET, B. et al. 1983b.The physiology of grass production under grazing. II Photosynthesis, crop growth and animal intake of continuously grazed sward. J. Appl. Ecology, 20(1):127-139.
PARSONS, A.J., PENNING, P.D. 1988. The effect of duration of regrowth on photosynthesis, leaf death and the average rate of growth in a rotationally grazed sward. Grass and Forage Sci., 43(1):15-27.

PILBEAM, C.J. 1992. Effect of nitrogen supply on the growth and senescence of leaves of Lolium perenne with contrasting rates of leaf respiration. Annals of Botany, 70(4):365-370.

POORTER, H. 1989. Interspecific variation in relative growth rate: on ecological causes and physiological consequences. In: Lambers, $\mathrm{H}$. et al. Causes and consequences of variation in growth rate and productivity of higher plants. Hague: Netherlands. p.45-67.

RADFORD, P.J. 1967. Growth analysis formulae: their use and abuse. Crop Sci., 7(3):171-175.

ROBSON, M.J. 1973. The growth and development of simulated swards of perennial ryegrass. II - Carbon assimilation and respiration in a seedling sward. Annals of Botany, 37(151):501-518.

ROBSON, M.J., RYLE, G.J.A., WOLEDGE, J. 1988. The grass plant - its form and function. In: Jones, M.B., Lazemby, A. The grass crop: The physiological basis of production. Chapman and Hall, NY. p.25-84.

SILSBURY, J.H. 1971. The effect of temperature and light energy on dry weight and leaf area changes in seedling plants of Lolium perenne L. Austr. J. Agric. Res., 22(2):177-187.

WILSON, G.L., LUDLOW, M.M. Net photosynthesis rates of tropical grass and legume leaves. In: NORMAN, M.J.T. (Ed.). Int. Grassland Congress, 11, 1970, Surfers Paradise Queensland. Proceedings... Austrália: CSIRO. p.534-538, 1970.

WOLEDGE, J., LEAFE, E.L. 1976. Single leaf and canopy photosynthesis in a ryegrass sward. Annals of Botany, 40(68):773-783.

WOLEDGE, J. 1978. The effect of shading during vegetative and reproductive growth on photosynthetic capacity of leaves in a grass sward. Annals of Botany, 42(181):1085-1089.
Recebido em: 02/07/98

Aceito em: 02/02/99 